\title{
BMJ Open Prospective daily diary study reporting of any and all symptoms in healthy adults in Pakistan: prevalence and response
}

Mudassir Anwar, ${ }^{1}$ James A Green, ${ }^{1}$ Pauline Norris, ${ }^{1}$ Nadeem Irfan Bukhari ${ }^{2}$

To cite: Anwar M, Green JA, Norris $\mathrm{P}$, et al. Prospective daily diary study reporting of any and all symptoms in healthy adults in Pakistan: prevalence and response. BMJ Open 2017;7:e014998. doi:10.1136 bmjopen-2016-014998

- Prepublication history and additional material for this paper are available online. To view these files, please visit the journal online (http://dx.doi. org/10.1136/bmjopen-2016014998).

Received 1 November 2016 Revised 8 September 2017 Accepted 4 October 2017

CrossMark

${ }^{1}$ School of Pharmacy, University of Otago, Dunedin, New Zealand ${ }^{2}$ University College of Pharmacy, University of the Punjab, Lahore, Pakistan

Correspondence to

Dr James A Green;

james.green@otago.ac.nz

\section{ABSTRACT}

Objectives Prevalence of symptoms in everyday life and how people respond to these symptoms is little studied outside Western culture and developed countries. We sought to use modified diary methods to explore the prevalence of and responses to symptoms in Pakistan. Design Prospective daily survey of symptoms and response.

Setting 8 cities across four provinces in Pakistan. Participants Stratified intercept in each city to recruit 153 participants of which 151 completed.

Primary and secondary outcome measures Each day for 30 days, participants were prompted by text message (short message service (SMS)) to complete a symptom diary. On days where symptoms were experienced, participants also reported how they responded. Prevalence was adjusted to population age and gender distributions.

Results $92 \%$ of participants experienced symptoms (adjusted prevalence $94 \%$, 95\% Cl $91 \%$ to $97 \%$ ), with musculoskeletal pain $(83 \%$, adj. $84 \%, 95 \% \mathrm{Cl} 84 \%$ to $90 \%)$ and respiratory symptoms $(75 \%$, adj. $77 \%, 95 \% \mathrm{Cl}$ $71 \%$ to $84 \%$ ) the most prevalent types of symptoms. Self-medication and use of home remedies and traditional medicines were the most common responses. Seeking professional help or using conventional medicine were less common, and self-medication responses included the use of antibiotics without prescription. The range of home remedies and traditional medicines was very diverse. Conclusions While symptom experience in Pakistan was similar to Western countries, home remedies were much more frequently used to respond to symptoms. Understanding how people respond and manage their experience of symptoms outside formal healthcare is important for designing effective policy and interventions, and this needs to be understood within the broader context including the cultural and economic setting, the health system and other structural determinants of health.

\section{INTRODUCTION}

Chronic conditions and symptoms of chronic conditions have been extensively studied, whereas many other symptoms remain neglected and poorly understood. In particular, symptoms are not often studied independent of disease states that are assumed to cause
Strengths and limitations of this study

- Our sample represents the geographical diversity across Pakistan.

- Using a prospective daily sampling method limits memory biases.

- Cultural restrictions prevent random sampling, which limits the representativeness of our sample.

them. However, symptoms in general are part of day-to-day life. They are often transient in nature, and may be ignored or treated outside formal healthcare. Some symptoms will go on to become indications of more serious illness, but understanding people's general symptom experience may shed light on how they determine which symptoms they come to interpret as requiring further treatment.

Data on experience of symptoms and how they are responded to is often collected during visits to clinics or hospitals. Such an approach misses a substantial proportion of the population and a considerable proportion of illness-any symptoms managed outside formal healthcare are missed. Further, physicians may define illness differently from their patients or from those patients who never or rarely consult physicians. Selection bias is then an important confounder in clinical-based studies as people coming to a healthcare facility are very likely different from those who do not. The study of minor symptoms as the cause of 'unnecessary' medical consultations ${ }^{12}$ provides a different but still biased lens through which to examine symptom experience. Community-based studies minimise these biases, but are few in number. ${ }^{3-5}$

A second methodological issue is that symptom data is most often collected retrospectively. ${ }^{5-7}$ Recall bias is a confounder in such studies as recall is affected by current state and experiences since the recalled event, as well as biases in memory. For low intensity 
or less severe events, as the recall period increases, the accuracy and completeness of the recalled event by the study participants decreases. In symptom research, retrospective reporting has been shown to underestimate the prevalence of symptoms in general, ${ }^{8}$ and musculoskeletal, digestive and nervous system symptoms in particular, relative to respiratory complaints such as colds. ${ }^{9}$ To deal with this difficulty, pen-based and paper-based diaries have sometimes been used to track symptoms. ${ }^{8}{ }^{10}$ However, doubts have been raised about both the accuracy and timing of such responses. ${ }^{11}$ Within this general diary framework, electronic devices have increasingly been used to collect data and provide reminders for self-report, including for symptom experience. ${ }^{12}$

A review of the existing literature on responses to symptoms found that most studies were conducted in developed countries. These include the USA, the UK, the Netherlands, New Zealand, Denmark and Switzerland, ${ }^{3-589}{ }^{12-14}$ with few in low-income and middle-income countries. ${ }^{15}$

People do not objectively experience symptoms, nor respond to them without context. In drawing together and integrating the main models of illness behaviour, Wyke and colleagues ${ }^{16}$ argue for a comprehensive and holistic model that involves the noticing of changed bodily experience, the interpretation of that changed experience as symptoms based on a person's knowledge and social network, and the culture and society in which they live. Responses are then based not just on the nature of the symptoms themselves and their daily impacts, but on social processes (within family and culture), on resources and the structure of the healthcare system, on interactions within the healthcare system and prior experiences with healthcare.

To remedy the lack of information about symptoms and response to symptoms in low-income and middle-income countries, selecting a strongly contrasting country best illustrates the extent to which information from developed countries can be applied to low-income and middle-income countries. Pakistan is a middle-income South Asian country with a population of 182.1 million (2013 estimate) ${ }^{17}$ It is predominantly Muslim, but has numerous ethnic minorities. No single low-income or middle-income country will be representative of all other low-income and middle-income countries, with Pakistan expected to strongly contrast with countries in Africa or South America, for example. But the extent to which patterns in Pakistan overlap with Western cultures presents some initial estimate of shared experience of symptoms and response.

The health system in Pakistan has both public and private sectors. Public sector services are provided at low cost. In the fee-for-service private sector, there are some accredited outlets and hospitals, but also many homoeopaths, traditional/spiritual healers, Greco-Arab healers, herbalists, bonesetters and unqualified practitioners. ${ }^{18}$

We could not locate a single study addressing people's day-to-day experience of symptoms and the way they respond to them in Pakistan. A recent review of health-seeking behaviour in Pakistan ${ }^{19}$ described mostly cross-sectional interview-based data. The recall period in most of the reviewed studies was not defined, making it difficult to determine the frequency with which symptoms and health-seeking occur. Themes emerging from the review were that: the private healthcare sector was preferred over the public sector; self-medication was fairly common; traditional and informal healers were heavily involved in the healthcare system; gender discrimination occurred, where women were sometimes prevented from getting or reluctant to get appropriate medical care; and there were certain myths and misconceptions related to disease and its treatment, for example, considering diarrhoea as a natural condition ${ }^{20}$ and applying onion over snake bite. ${ }^{21}$ Following the publication of that review, research has continued to focus on specific population groups (eg, pregnant women ${ }^{22}$ ).

To prospectively explore symptom experience and response in Pakistan, we used an adapted version of modern electronic diary methods, adapted to fit around the practical constraints of a middle-income country. The current study aimed to quantify the frequency and type of symptoms experienced by healthy individuals in daily life in Pakistan, and the frequency and type of responses to those symptoms.

\section{METHODS \\ Design}

This study used a longitudinal diary approach, collecting experience of symptoms and responses to symptoms every 24 hours for 30 days. More frequent (ie, several times a day) symptom data has been collected but only for a week. ${ }^{12}$ Collecting data only once a day enabled us to gain a broader picture of symptom experience and response over a longer period. We considered that more frequent sampling would have been too intensive for the participants.

To the best of our knowledge, modern diary methods have not been used in low-income or middle-income countries or in non-Western cultures. Modern diary methods often use electronic reminders, ${ }^{23}$ but the electronic platforms used to support them may be less available or functional in low-income and middle-income countries. In Pakistan, mobile phone penetration is high, but internet access and mobile internet access are limited. Further, Urdu, the national language of Pakistan, uses an alphabet that is not well supported, especially on mobile platforms. The advantages of electronic reminders and data collection are that they provide a clear reminder to participants, and also minimise the likelihood that surveys will be retrospectively filled in later. The best compromise was, therefore, to revert to a more traditional paper diary, but to send participants a daily short message service (SMS) (text message) at a time of their choosing to serve as a reminder. Further, they were provided with envelopes so that they could send their diaries back weekly to further enforce time compliance. Though the participants were not expected to respond to the SMS reminders, most of 
Table 1 Geographical distribution of sample recruited in Pakistan (2012-2013)

\begin{tabular}{lllll}
\hline Province & Proportion of population \% & Proportion of sample \% (n) & City (within province) & Participants (n) \\
\hline Punjab & 57 & $57(87)$ & Lahore & 62 \\
& & & Jhelum & 15 \\
& & & Wah Cantt & 10 \\
\hline Sindh & 24 & $24(37)$ & Karachi & 25 \\
& & & Sukkur & 12 \\
Khyber Pakhtunkhwa & 14 & $14(22)$ & Peshawar & 15 \\
Balochistan & 5 & $5(7)$ & Dir & 7 \\
\hline
\end{tabular}

$\mathrm{n}=153$ (two participants did not complete the 30 days of symptom reporting).

them confirmed once they had filled the questionnaire. Thus, although some research has shown audio reminders to fill diaries to not be particularly effective,${ }^{24}$ SMS may be more effective as a target reminder. ${ }^{25} 26$

\section{Population and sampling}

We planned to recruit 150 healthy members of the general public aged 18-65, from eight cities from four provinces of Pakistan based on population density. One city from each province was the provincial capital, also representing larger cities, whereas the other cities selected were smaller and not so developed. Punjab being the most populous province contributed three cities whereas Balochistan being the least populated contributed only the provincial capital. Table 1 presents the geographical distribution of the sample in Pakistan.

Cultural norms in Pakistan mean that women are less likely to answer the telephone, and it is not normal for people to communicate with strangers, especially of the opposite gender. ${ }^{27}$ This meant that most methods of recruiting a probability sample such as random digit dialling would not work. Because we could not use a conventional probability sample, the most feasible sampling solution was recruiting participants in places known to differ systematically, both within a city and across the country, to get a more representative and diverse sample. We refer to this method as a stratified intercept sample. In each city, we used one man and one woman, each recruiting members of the same gender from places such as markets, parks, public and private offices and universities. Some research assistants may have also recruited some participants via their own social networks. Although we had hoped to include people with poor literacy, hoping that household members, for example, might help them out, from subsequent discussions with research assistants and the demographic profile of our data, it is clear that we recruited only literate participants. We targeted a sample size of 150 participants due to limits on budget and time.

\section{Procedure}

The data was gathered from December 2012 to February 2013, with the help of 11 research assistants (six men and five women) each based in one of the cities included in the study. The instruments and the methods were piloted on 10 individuals prior to starting data collection.

Participants completed an initial face-to-face session where they were recruited: they completed a series of questionnaires including demographic information, beliefs about medicines, time preference and attitudes towards different health professionals. They were then briefed on the daily symptom reporting procedure, and received 30 copies of the symptom reporting questionnaire and four self-addressed and stamped envelopes.

The participants completed the first page of the symptom reporting questionnaire daily and the rest of the questionnaire only on the days when they experienced one or more symptoms. A text message reminder to fill the questionnaire was sent daily at the time they indicated was best for them. In order to minimise the chances of retrospective filling of the questionnaires, participants were instructed to post back the questionnaires weekly using postpaid return-addressed envelopes provided to them. A reminder to do so was sent out to the participants on days 7, 14, 21 and 30. At the end of 30-day period, each participant received Rs 1000 ( US\$9.80) in cash as a token of appreciation for their time.

\section{Materials}

The daily symptom reporting questionnaire gathered information on the type, severity and duration of symptoms and responses to symptoms experienced by the study participants. Participants were first asked to rate their happiness and wellness on a scale of 1-10 and if they experienced any symptoms during the last 24 hours. The list of possibly experienced symptoms was informed by qualitative interviews and focus groups. ${ }^{28}$ The 'daily health record' ${ }^{3}$ was used as a model to design the portion of the questionnaire that focused on responses to symptoms, with additional responses from the interviews and focus groups. The Urdu questionnaire, along with its English translation are available in the online supplementary materials.

All materials were developed in English and back-translated to Urdu, following WHO guidelines. ${ }^{29}$ Initial translation into Urdu was performed by the first author (a native speaker), which was then discussed with the fourth 
author (also a native speaker). Two independent bilingual translators (and native speakers of Urdu) translated it back to English, and the original and back-translated version were then compared by the second and third authors (native-English speakers). Observed differences were discussed to a consensus solution with the translators. These changes were incorporated and then pretested on a group of five respondents from Pakistan. No major issues were detected in terms of understanding of the questions and conceptual equivalence, but some minor changes were made.

\section{Analysis}

R V.3.3.1/R Studio V.0.99.902 was used for data analysis. One-month prevalence was calculated as the proportion of participants experiencing a symptom at least once over the month. Prevalence is also presented with symptoms grouped into: (1) respiratory, (2) pain, (3) gastrointestinal, (4) low energy (including fever and tiredness) and (5) other. Prevalence was adjusted to match 2012-2013 age and gender estimates of Pakistan's population, ${ }^{30}$ using the rake and trimweight functions in the survey package. ${ }^{31}$ Weights were trimmed to 0.3 and 3 to ensure that no individuals are unduly overweighted or underweighted. For each symptom (or symptom group) the number of days that symptom was experienced was averaged for participants experiencing that symptom, adjusting for age and gender (eg, participants that experienced cough did so on average 3.4 days).

The seven possible responses to symptoms included in the symptom reporting questionnaire were: change in routine, talking to someone about symptoms, trying home remedies, using medicine, considering seeking help from a healthcare professional, actually seeing a healthcare professional, and ignoring the symptoms or taking no action. Some 'other' actions were recoded into these categories as appropriate. 'Using medicine' was recoded into new variable 'self-medication' by ascertaining if the medicine was not prescribed by the doctor or purchased from a pharmacist during the previous days or available only on prescription. Other instances considered self-medication were those where the medicine was purchased from a pharmacy but not from the pharmacist. Prevalence and mean number of responses for participants making that response were again adjusted for age and gender. Adjusted logistic regression (using svyglm in the survey package) was used to determine whether the number of days on which symptoms where experienced predicted making each response.

\section{RESULTS}

\section{Participants}

Demographic characteristics are presented in table 2. Mean age was 28.4 years (range: $18-61$ ) and 54\% were men. The largest ethnic group was Punjabi (42\%), consistent with the fact that Punjab province is the most populous, and home to the Punjabi ethnic group. The sample was relatively highly educated, with a high proportion of professionals and students.

\section{Symptom reporting}

Out of 153 participants recruited, 151 completed the 30-day period. One week of questionnaires from two participants went missing in the post. There was one case where a participant posted the next week's questionnaires along with the previous weeks'. It is possible that participants who were generally healthy and had a few consecutive days without symptoms might have filled some of the questionnaires in advance as they might not be expecting any symptoms in near future. However, as previously noted, most participants responded to the SMS to indicate that they had filled out the questionnaire.

There were 1475 symptomatic days reported by participants, but the questionnaire was completely filled for 1469 days. Thus, there were 6 days ( $0.4 \%$ of the total days) when the questionnaire was incompletely filled by four participants. Taken together with the apparent low rate of retrospective filling out of the questionnaire, it seems that our adapted diary methods can produce good quality data in middle-income countries.

\section{Prevalence and frequency of symptoms}

One hundred and forty-two participants (92\%, adjusted prevalence $94 \%, 95 \%$ CI $91 \%$ to $97 \%$ ) reported at least one symptom during the 30-day period. The number of days on which a symptom was experienced ranged from 0 to 30, with an average of 9.7 (adj. 10.6, 95\% CI 9.2 to 12.0) days per study participant and 10.4 (adj. 11.2, 95\% CI 9.8 to 12.7) days per symptomatic participant.

The modal number of symptom types was tied between three and seven (14\% each of the sample) followed by $12 \%$ who reported two. Total symptom burden-counting the number of symptoms experienced across participantsranged from 0 to 44, with an average of 11.7 (adj. 12.8, 95\% CI 10.7 to 14.9) for all participants and 12.4 (adj. 13.5, $95 \%$ CI 11.4 to 15.7 ) for symptomatic participants.

As the left-hand panels of figure 1 show, headache was the most prevalent symptom, followed by sore throat and cough. Following this pattern, pain was the most frequent symptom group, followed by respiratory.

In terms of the number of days, a symptom was experienced on average for participants experiencing that symptom, allergy, cough and headache had the highest number of days (right-hand panels of figure 1). The maximum number of days a symptom was experienced by any of the participants was a cough, reported on 27 days by one of the participants, followed by headache (18 days) and back/neck pain (12 days).

\section{Response to symptoms}

Changing from normal routine, talking to someone about their symptoms, home remedies and self-medication were all very common choices to deal with symptoms (figure 2 ). More than half of the participants considered seeking help while half of the participants actually sought help. Ignoring the symptoms was the least common response. Talking to someone and changing from normal routine 
Table 2 Demographic characteristics of the sample (Pakistan, 2012-2013)

\begin{tabular}{lll}
\hline Characteristic & n (total=151) & $\%$ \\
\hline Gender & & \\
Male & 82 & 54 \\
Female & 69 & 46
\end{tabular}

Ethnicity

\begin{tabular}{lrr} 
Punjabi & 64 & 42 \\
\hline Urdu speaking/Mohajir & 47 & 31 \\
\hline Pakhtun/Pathan & 23 & 15 \\
\hline Baloch & 6 & 4 \\
\hline Sindhi & 4 & 3 \\
\hline Saraiki & 4 & 3 \\
Hindko & 2 & 1 \\
Other & 1 & 1
\end{tabular}

\begin{tabular}{lrr}
\hline Age (years) & & \\
\hline Young adults (18-34) & 126 & 83 \\
\hline Middle age group (35-54) & 22 & 15 \\
\hline $\begin{array}{l}\text { Older adults (55-65) } \\
\text { Education level }\end{array}$ & 3 & 2 \\
$\begin{array}{l}\text { Low (up to 8years of formal } \\
\text { education) }\end{array}$ & 15 & 10 \\
\hline
\end{tabular}

\begin{tabular}{lrr}
$\begin{array}{l}\text { Middle (up to } 12 \text { years of formal } \\
\text { education) }\end{array}$ & \\
$\begin{array}{l}\text { Matric/secondary (10 years of formal } \\
\text { education) }\end{array}$ & 20 & 13 \\
$\begin{array}{l}\text { Diploma/certificate } \\
\text { Intermediate/higher secondary }\end{array}$ & 5 & 3 \\
\hline
\end{tabular}

(12 years of formal education)

$\begin{array}{lrr}\text { High } & & \\ \text { Tertiary bachelor's degree } & 41 & 27 \\ \text { Master's degree } & 41 & 27 \\ \text { Doctoral degree } & 5 & 3\end{array}$

\begin{tabular}{|lrr}
\hline Occupation & 11 & 7 \\
\hline Managers & 35 & 23 \\
\hline Professionals & 7 & 5 \\
\hline Technicians/trade workers & & 7 \\
\hline $\begin{array}{l}\text { Service workers and shop and market } \\
\text { sale workers }\end{array}$ & 10 & 3 \\
\hline Clerical and administrative workers & 5 & 3 \\
\hline Crafts and related trades workers & 5 & 1 \\
\hline Machinery operators/drivers & 2 & 8 \\
\hline $\begin{array}{l}\text { Elementary unskilled professions/ } \\
\text { labourers }\end{array}$ & 12 & \\
\hline Students & 33 & 22 \\
\hline Housewives & 11 & 7 \\
\hline $\begin{array}{l}\text { Not employed/not working for pay/ } \\
\text { jobless/retired }\end{array}$ & 6 & 4 \\
\hline Did not respond & 14 & 9 \\
\hline
\end{tabular}

Continued
Table 2 Continued

\begin{tabular}{lcr}
\hline Characteristic & n (total=151) & \% \\
\hline Pregnant & & \\
Yes & 1 & 1 \\
No/not applicable & 150 & 99 \\
\hline
\end{tabular}

were the two most frequently used responses. Increasing days on which symptoms were experienced predicted all responses except changing from normal routine (OR 1.23 (95\% CI 0.93 to 1.63 ); home remedy use, OR 1.22 (1.09-1.36); self-medication OR 1.52 (1.28-1.80), considering visiting a health professional, OR 1.22 (1.13-1.32), visiting a health professional, OR 1.15 (1.08-1.23), other actions, OR $1.10(1.02-1.19)$ and taking no action, OR $1.07(1.02-1.12))$.

\section{Types of home remedies, self-medication and other actions}

Online supplementary table 1 contains a wealth of detail on specific home remedies, medicines/self-medication and other actions broken down by symptoms. Home remedies, including food items, herbs and condiments available at home, were used to alleviate symptoms. Some of the most common were: tea (with or without milk), egg, milk, honey, green tea, fruits, ginger and spices such as fennel, Ajwain seeds (Carom or Bishop Weed; Trachyspermum ammi) and clove.

Paracetamol alone or in combination was the most common form of self-medication. Though less common, a concerning finding was the use of a variety of antibiotics including metronidazole (15 mentions), amoxicillin (14), levofloxacin (4), erythromycin (3), ampicillin (1) and azithromycin (1).

'Other' responses included massage, topical balms, exercise and heat and cold therapy to defeat pain symptoms. Prayers and recitation were also reported.

\section{Type of help considered and sought}

Doctors were the health professionals that participants most commonly considered contacting and most commonly visited (table 3 ). The next most frequently considered and visited health professionals were homoeopaths, medical store staff and pharmacists.

\section{Social networks and response to symptoms}

Social networks were heavily involved in making choices about how to respond to symptoms (table 4). Other household members were most often involved in these decisions, followed by discussions with a spouse and then a friend, neighbour or colleague.

\section{DISCUSSION}

Symptoms in daily life were very prevalent in Pakistan. Direct comparison with previous findings is difficult because of the differences in methodological approaches which may mask or enhance genuine cultural differences. Nonetheless, the findings are consistent with those of the 


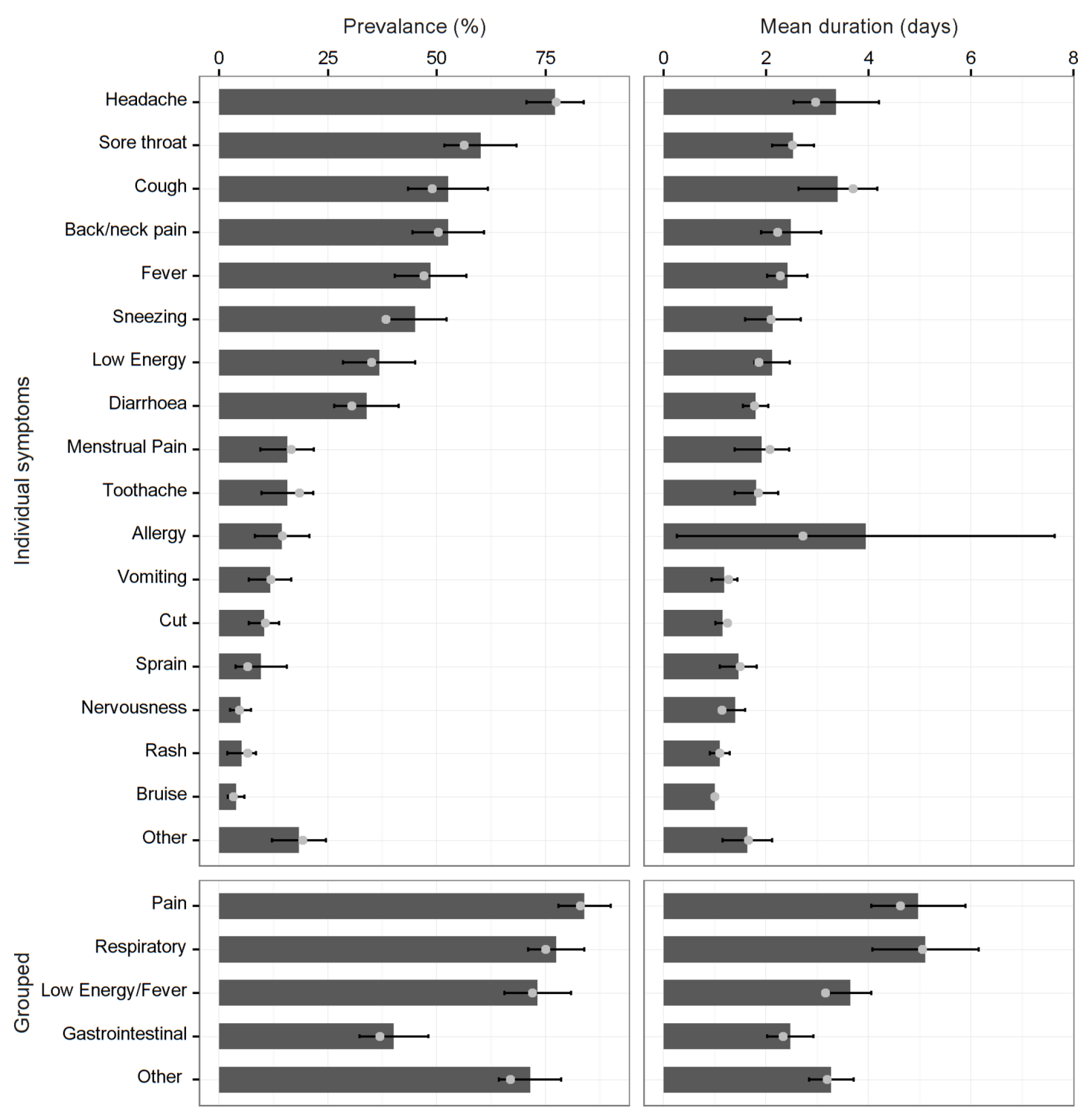

Figure 1 Prevalence of symptoms and symptom groups adjusted for age and gender in Pakistan (left panels); and adjusted mean duration of a symptom for people experiencing that symptom/symptom group (right panels). Error bars represent $95 \%$ Cls (allergy symptoms were experienced by a small number of participants, with higher variability producing a noticeably wider $\mathrm{Cl})$. Grey points represent unadjusted values.

previous large-scale studies in Western countries with headache, body/neck pain, respiratory symptoms (such as cough, cold, sore throat) and the feeling of tiredness/ low energy being some of the most commonly reported symptoms. 341332

Only two previous studies have reported on the frequency of symptoms separately from prevalence. Verbrugge and Ascione ${ }^{10}$ found respiratory symptoms to be the most frequent, whereas Brody and Kleban ${ }^{33}$ found pain days accounted for $38 \%$ of the total symptom days in older adults. We found pain to be the most frequent. Understanding the frequency with which different types of symptoms occur separate from prevalence is important in understanding the daily burden of different types of symptoms.

Ignoring symptoms is a common response in other studies in Western countries, ${ }^{34}{ }^{35}$ but this was not the case in Pakistan, where there may be a lower awareness of the self-limiting nature of some symptoms. Furthermore, the pluralistic healthcare system in Pakistan provides many easily accessible options, which might increase the rate of active responses to symptoms. These active responses became more likely as the number of days a symptom was experienced increased.

Self-care was the dominant response. Changing routine, getting advice from lay people on symptoms and/or treatment, trying a home remedy and self-medicating, all come under the umbrella of self-care.

Self-medication is an integral part of self-care. Participants' high rate of self-medication is consistent with a number of other studies in both Pakistan ${ }^{62136-38}$ and other countries. ${ }^{10} 3940$ Over-the-counter medicines, especially pain killers, were some of the most commonly used medicines by the participants. Urquhart $e t a l^{41}$ reported 


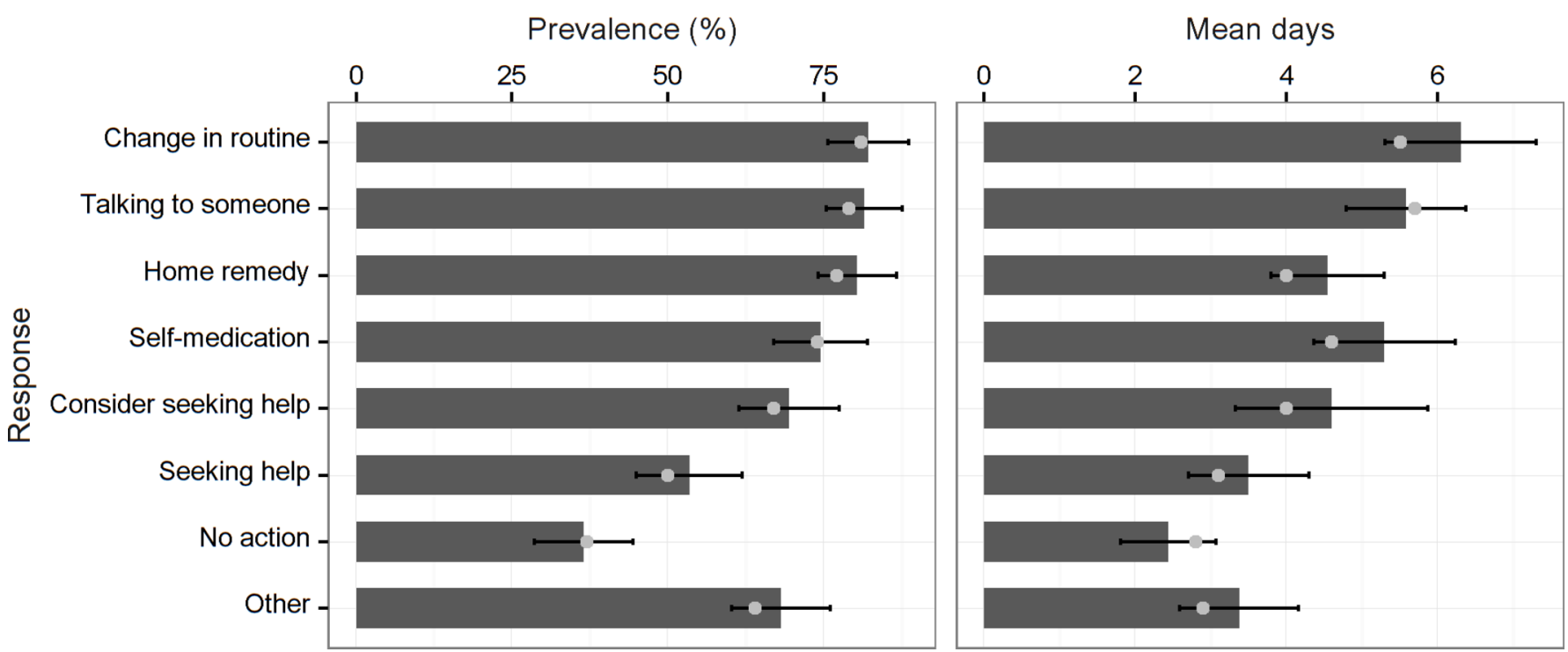

Figure 2 Prevalence of responses to symptoms adjusted for age and gender in Pakistan (left panel); and adjusted mean number of days on which that action was taken in relation to that symptom (right panel). Error bars represent $95 \% \mathrm{Cls}$. Grey points represent unadjusted values.

the use of over-the-counter medicines by nearly half of the patients seeing a general practitioner in Scotland. However, participants in this study also reported using prescription-only medicines without consulting a qualified healthcare professional. Participants self-medicated for all kinds of conditions-whether transient or long term-and with all kinds of medicines. Social networks also played a role in treatment decisions, potentially increasing lay diagnosis and medication.

A concerning finding of this study is the use of antibiotics without consultation. One of the prominent factors that influence such practices is 'drug retail-shops' commonly known as 'medical stores', being the public's first point of contact with the healthcare system. ${ }^{42}$ In Pakistan, having a prescription to buy a medicine is not necessary, as the existing legal restrictions are not implemented. ${ }^{43}$ Reasons

Table 3 Number of times seeking help was considered and actually sought (Pakistan, 2012-2013)

\begin{tabular}{lcl}
\hline & $\begin{array}{l}\text { Number of days } \\
\text { consultation } \\
\text { was considered }\end{array}$ & $\begin{array}{l}\text { Number } \\
\text { of days } \\
\text { consultation } \\
\text { was actually } \\
\text { sought }\end{array}$ \\
\hline Healthcare professional & 152 \\
\hline Doctor & 257 & 18 \\
\hline Homoeopath & 45 & 31 \\
\hline Medical store staff & 42 & 30 \\
\hline Pharmacist & 37 & 6 \\
\hline Dentist & 20 & 4 \\
\hline Herbalist & 14 & 5 \\
Spiritual healer & 7 & 3 \\
\hline Bone setter & 6 & 3 \\
\hline Physiotherapist & 3 & \\
\hline
\end{tabular}

for this include: it being a common practice in Pakistan, the higher cost of other treatments, extra cost of doctors, long waiting times, doctors not being available 24 hours a day, doctors' knowledge not being up to standard, confidence in their own knowledge of medicine, and pharmacists being a more up-to-date source of knowledge about drugs. ${ }^{44}$

The scarcity of data on over-the-counter misuse and the benefits of product availability should be balanced against the risk of misuse. ${ }^{41}$ This study provides a window into how and what type of over-the-counter medicines are used. Paracetamol and other pain killers were the most common. There is a strong evidence of antibiotic misuse in a variety of countries ${ }^{45-47}$ and it is now clear that this is also an issue in Pakistan.

Home remedies are one of the least studied types of selfcare. ${ }^{48} 49$ Home remedies are the first step in responding to illness in Pakistan, ${ }^{60}$ confirmed by this study. This underscores the importance of further research focusing on exploring the benefits and the potential risks, and interactions of home remedies if they are consumed alongside or in place of conventional medicines.

Traditional medicines were also frequently used to alleviate minor symptoms. The study reported the use of Joshanda (a commercially available herbal tea, typically containing Glycyrrhiza glabra, Justicia adhatoda, Hyssopus officinalis, Camellia sinensis, Ephedra sinica, Mentha $x$ piperita, Foeniculum vulgare and Eucalyptus globulus) as one of the most commonly used traditional medicine to alleviate respiratory symptoms which is consistent with other findings. 50

Seeking formal help for symptoms was among the least common responses. As discussed earlier, studies on responses to symptoms have often focused on symptom reporting to healthcare professionals. However, the current study suggests that reporting symptoms to 
Table 4 Number of times social networks were contacted in responding to symptoms (Pakistan, 2012-2013)

\begin{tabular}{|c|c|c|c|c|}
\hline \multirow[b]{2}{*}{ Response } & \multicolumn{4}{|c|}{ Social network } \\
\hline & Spouse & $\begin{array}{l}\text { Other members of } \\
\text { household }\end{array}$ & Other relatives & $\begin{array}{l}\text { Friend/colleague/ } \\
\text { neighbour }\end{array}$ \\
\hline Talking to someone about symptoms & 129 & 374 & 40 & 205 \\
\hline Trying home remedies & 42 & 144 & 18 & 34 \\
\hline Self-medication & 28 & 153 & 10 & 40 \\
\hline Seeking help & 3 & 27 & 5 & 12 \\
\hline 'Other' action & 19 & 56 & 5 & 12 \\
\hline
\end{tabular}

healthcare professionals is just one of many possibilities, and that in Pakistan, it is the least common one. Thus, any study that recruits participants when they attend formal healthcare will miss most symptoms and most responses to these symptoms.

Doctors were the first choice for health professional advice. Pharmacists, despite being an obvious choice for minor symptoms in high-income countries, were not a common choice for seeking help from in Pakistan. This might be because the informal sector accounts for more than $70 \%$ of the consultations in Pakistan. ${ }^{51}$ Healer shopping is a common practice in Pakistan as people tend to change healers often because they want quick results. ${ }^{650}$

We are aware of a number of limitations of this study. First, by standards expected of research conducted in high-income countries, we have produced what could be described as a convenience sample. However, the methods used in high-income countries, such as calling people using random digit dialling $\left(\mathrm{eg},{ }^{5}\right)$ are not possible in some low-income and middle-income countries, as outlined earlier. This research, therefore, should provide the best available estimation of prevalence and frequency of symptoms experienced in Pakistan. Second, the findings are based on self-reporting of symptoms and responses, which might have been affected by exaggeration or under-reporting of certain events. However, according to Kroenke and Price,$^{32}$ patients are the best judge of symptoms. Third, as the study recorded the experiences of people mostly in the cooler season, it might have resulted in over or under-representation of certain symptoms. However, due to time constraints, it was not practical to conduct the study for a month in each season or for a full year. Fourth, the findings of this study sample represent mainly the urban population of Pakistan, which may limit its generalisability to rural areas. However, a strength is that we recruited people from metropolitan cities and from smaller cities and a remote city in Khyber Pakhtunkhwa (Dir). Finally, although the health diary offers numerous advantages over other methods, it introduces different types of bias. Notably, its literacy requirements limit our generalisability to more literate populations in Pakistan. This largely overlaps with our restriction to urban areas, which have a much higher adult literacy rate $(74 \%)$ compared with rural areas $(47 \%){ }^{52}$ There is also a gender gap in literacy, with rates for men around $15 \%$ higher than women in both urban and rural areas. Filling out a health diary, also may encourage participants to pay more attention to their symptomatic experience, which may increase the number of symptoms reported.

\section{CONCLUSIONS}

Symptoms are prevalent in Pakistan, with musculoskeletal pain and respiratory symptoms the most common. Self-care was the main response, frequently including home remedies and talking to someone about the symptoms. Participants are also self-medicated, including potentially harmful medicines such as antibiotics without being prescribed by a doctor. Where participants sought help, doctors and informal healers were most frequently consulted.

Acknowledgements We acknowledge the contribution made by the research assistants with the collection and entry of data.

Contributors JAG generated the initial study idea. MA, JAG, PN and NIB designed the study and drafted the manuscript. MA collected the data, under the oversight of JAG, PN, NIB. JAG and MA performed the data analysis. All authors contributed with the interpretation of the results. JAG is the guarantor of the study.

Competing interests None declared.

Ethics approval In Pakistan, ethical approval was received from the University of the Punjab (HEC/UCP/1916A). We also received approval from the University of Otago Human Ethics Committee (F12/008). All participants provided written consent.

Provenance and peer review Not commissioned; externally peer reviewed.

Data sharing statement Data files for analysis are archived on the Open Science Framework's website (https://osf.io/y86xa/).

Open Access This is an Open Access article distributed in accordance with the Creative Commons Attribution Non Commercial (CC BY-NC 4.0) license, which permits others to distribute, remix, adapt, build upon this work non-commercially, and license their derivative works on different terms, provided the original work is properly cited and the use is non-commercial. See: http://creativecommons.org/ licenses/by-nc/4.0/

(c) Article author(s) (or their employer(s) unless otherwise stated in the text of the article) 2017. All rights reserved. No commercial use is permitted unless otherwise expressly granted.

\section{REFERENCES}

1. Cantrill J, Morris C, Weiss M. How patients perceive minor illness and factors influencing seeing a doctor. Primary Health Care Research and Development 2006;7:157-64.

2. Vedsted P, Fink P, Sørensen HT, et al. Physical, mental and social factors associated with frequent attendance in Danish general 
practice. A population-based cross-sectional study. Soc Sci Med 2004:59:813-23.

3. Verbrugge LM. Triggers of symptoms and health care. Soc Sci Med 1985:20:855-76.

4. Wyke S, Hunt K, Ford G. Gender differences in consulting a general practitioner for common symptoms of minor illness. Soc Sci Med 1998;46:901-6.

5. Petrie KJ, Faasse K, Crichton F, et al. How common are symptoms? Evidence from a New Zealand national telephone survey. BMJ Open 2014;4:e005374.

6. Hunte PA, Sultana F. Health-seeking behavior and the meaning of medications in Balochistan, Pakistan. Soc Sci Med 1992;34:1385-97.

7. Elnegaard S, Pedersen AF, Sand Andersen R, et al. What triggers healthcare-seeking behaviour when experiencing a symptom? Results from a population-based survey 2017.

8. Kooiker SE. Exploring the iceberg of morbidity: a comparison of different survey methods for assessing the occurrence of everyday illness. Soc Sci Med 1995;41:317-32.

9. Marcus AC. Memory aids in longitudinal health surveys: results from a field experiment. Am J Public Health 1982;72:567-73.

10. Verbrugge LM, Ascione FJ. Exploring the iceberg-common symptoms and how people care for them. Med Care 1987;25:539-69.

11. Stone AA, Shiffman S, Schwartz JE, et al. Patient compliance with paper and electronic diaries. Control Clin Trials 2003;24:182-99.

12. Michel G. Daily patterns of symptom reporting in families with adolescent children. Br J Health Psychol 2007;12:245-60.

13. McAteer A, Elliott AM, Hannaford PC. Ascertaining the size of the symptom iceberg in a UK-wide community-based survey. British Journal of General Practice 2011;61:1-11.

14. Elnegaard S, Andersen RS, Pedersen AF, et al. Self-reported symptoms and healthcare seeking in the general population-exploring "The Symptom Iceberg". BMC Public Health 2015;15:685.

15. Leyva-Flores R, Luz Kageyama M, Erviti-Erice J. How people respond to illness in Mexico: self-care or medical care? Health Policy 2001;57:15-26.

16. Wyke S, Adamson J, Dixon D, et al. Consultation and illness behaviour in response to symptoms: a comparison of models from different disciplinary frameworks and suggestions for future research directions. Soc Sci Med 2013;86:79-87.

17. World Bank, Pakistan Data Page. 2014. http://data.worldbank.org/ country/pakistan.

18. Shaikh BT, Haran D, Hatcher J, et al. Studying health-seeking behaviours: collecting reliable data, conducting comprehensive analysis. J Biosoc Sci 2008;40:53-68.

19. Anwar M, Green J, Norris P. Health-seeking behaviour in Pakistan: a narrative review of the existing literature. Public Health 2012;126:507-17.

20. Mull JD, Mull DS. Mothers' concepts of childhood diarrhea in rural Pakistan: what ORT program planners should know. Soc Sci Med 1988;27:53-67.

21. Chandio AM, Sandelo P, Rahu AA, et al. Snake bite: Treatment seeking behaviour among Sindh rural population. Journal of Ayub Medical College 2000;12:3-5.

22. Qureshi RN, Sheikh S, Khowaja AR, et al. Health care seeking behaviours in pregnancy in rural Sindh, Pakistan: a qualitative study. Reprod Health 2016;13:34.

23. Gunthert KC, Wenze SJ. Daily diary methods. In: Conner TS, Mehl MR, eds. Handbook of Research Methods in Daily LIfe. NY: Guilford Press, 2012:144-59.

24. Broderick JE, Schwartz JE, Shiffman S, et al. Signaling does not adequately improve diary compliance. Ann Behav Med 2003;26:139-48.

25. Bolger N, Davis A, Rafaeli E. Diary methods: capturing life as it is lived. Annu Rev Psychol 2003;54:579-616.

26. Ashby R, Turner G, Cross B, et al. A randomized trial of electronic reminders showed a reduction in the time to respond to postal questionnaires. J Clin Epidemiol 2011;64:208-12.

27. Paul J. International marketing: text and cases. New Delhi: Tata McGraw-Hill Education, 2008.
28. Anwar M, Green JA, Norris P, et al. Self-medication, home remedies, and spiritual healing: common responses to everyday symptoms in Pakistan. Health Psychol Behav Med 2015;3:281-95.

29. World Health Organisation. Process of translation and adaptation of instruments, 2010. http://www.who.int/substance_abuse/research_ tools/translation/en/.

30. Pakistan Bureau of Statistics. 2013. Labour Force Survey 2012-13. http://www.pbs.gov.pk/content/labour-force-survey-2012-13-annualreport.

31. Lumley T. Survey: analysis of complex survey samples: R package version 3. Journal of Statistical Software 2014;9.

32. Kroenke K, Price RK. Symptoms in the community. Prevalence, classification, and psychiatric comorbidity. Arch Intern Med 1993;153:2474-80.

33. Brody EM, Kleban MH. Day-to-day mental and physical health symptoms of older people: a report on health logs. Gerontologist 1983;23:75-85.

34. Stoller EP, Forster LE, Portugal S. Self-care responses to symptoms by older people. A health diary study of illness behavior. Med Care 1993;31:24-42.

35. Taylor J. Factors that influence the public's ability to self-medicate. Part 2-actions in seeking relief from minor illness. SelfCare Journal 2011;2:98-110.

36. Haider S, Thaver IH. Self medication or self care: implication for primary health care strategies. J Pak Med Assoc 1995;45:297-8.

37. Sadiq H, Muynck AD. Health care seeking behavior of pulmonary tuberculosis patients visiting TB Center Rawalpindi. J Pak Med Assoc 2001;51:10-16.

38. Shaikh BT, Haran D, Hatcher J. Where do they go, whom do they consult, and why? Health-seeking behaviors in the northern areas of Pakistan. Qual Health Res 2008;18:747-55.

39. Freer CB. Self-care: a health diary study. Med Care 1980;18:853-61.

40. Wijesinghe PR, Jayakody RL, de A Seneviratne R. Prevalence and predictors of self-medication in a selected urban and rural district of Sri Lanka. WHO South East Asia J Public Health 2012;1:28-41.

41. Urquhart G, Sinclair HK, Hannaford PC. The use of non-prescription medicines by general practitioner attendees. Pharmacoepidemiol Drug Saf 2004;13:773-9.

42. Kafle KK, Madden JM, Shrestha AD, et al. Can licensed drug sellers contribute to safe motherhood? A survey of the treatment of pregnancy-related anaemia in Nepal. Soc Sci Med 1996;42:1577-88.

43. Arshad MS, ljaz M, Hussain A. Evaluation of antibiotic use behavior in cold and flu amongst the students of Bahauddin Zakariya University Multan Pakistan. Pak. J. Pharm 2007;2010(20-23):15-22.

44. Rao MH, Soomro IBM. Attitude and practice pattern of urban population in the use of local pharmacy in treatment seeking process and it's comparison with the semi urban population of Karachi. Pak. J. Med. Res 2004:43:1-9.

45. Dameh M, Green J, Norris P. Over-the-counter sales of antibiotics from community pharmacies in Abu Dhabi. Pharm World Sci 2010;32:643-50.

46. Grigoryan L, Haaijer-Ruskamp FM, Burgerhof JG, et al. Selfmedication with antimicrobial drugs in Europe. Emerg Infect Dis 2006;12:452-9.

47. Mainous AG, Diaz VA, Carnemolla M. Factors affecting Latino adults' use of antibiotics for self-medication. J Am Board Fam Med 2008;21:128-34.

48. Chibwana Al, Mathanga DP, Chinkhumba J, et al. Socio-cultura predictors of health-seeking behaviour for febrile under-five children in Mwanza-Neno district, Malawi. Malar J 2009;8:219.

49. Ritchie JE, Herscovitch F, Norfor JB. Beliefs of blue collar workers regarding coronary risk behaviours. Health Educ Res 1994;9:95-103.

50. Kundi MZ, Anjum M, Mull DS, et al. Maternal perceptions of pneumonia and pneumonia signs in Pakistani children. Soc Sci Med 1993;37:649-60.

51. Karim M, Mahmood M. Health systems in Pakistan: a descriptive analysis. Karachi, Pakistan: Department of Community Health Sciences, Aga Khan University, 1999.

52. Pakistan Bureau of Statistics. Pakistan Social And Living Standards Measurement Survey 2012-13, 2013. http://www.pbs.gov.pk/ content/pakistan-social-and-living-standards-measurement-surveypslm-2012-13-provincial-district. 
Correction: Prospective daily diary study reporting of any and all symptoms in healthy adults in Pakistan: prevalence and response

Anwar M, Green JA, Norris P, et al. Prospective daily diary study reporting of any and all symptoms in healthy adults in Pakistan: prevalence and response. BMJ Open 2017;7:e014998. doi: 10.1136/bmjopen-2016-014998.

The author Mudassir Anwar's affiliation should be 'Al Ain University of Science and Technology, Al Ain, United Arab Emirates'. The work was conducted in University of Otago but at the time of submission Mudassir Anwar was working in his current work institution, $\mathrm{Al}$ Ain University of Science and Technology.

Open access This is an open access article distributed in accordance with the Creative Commons Attribution Non Commercial (CC BY-NC 4.0) license, which permits others to distribute, remix, adapt, build upon this work non-commercially, and license their derivative works on different terms, provided the original work is properly cited and the use is non-commercial. See: $\mathrm{http} / / / \mathrm{creativecommons.org/licenses/by-nc/4.0/}$

(c) Article author(s) (or their employer(s) unless otherwise stated in the text of the article) 2018. All rights reserved. No commercial use is permitted unless otherwise expressly granted.

BMJ Open 2018;8:e014998corr1. doi:10.1136/bmjopen-2016-014998corr1

Check for updates 